\title{
On the boundedness of the Weyl transform for homogeneous distributions on $\mathbb{R}^{2}$
}

\author{
Peter Wagner ${ }^{1}$
}

Received: 11 November 2019 / Revised: 24 February 2020 / Accepted: 29 February 2020 /

Published online: 13 March 2020

(c) The Author(s) 2020

\begin{abstract}
Starting from earlier work by M.W. Wong, we give necessary and sufficient criteria, respectively, for the boundedness of the Weyl transform $W_{\sigma}$ as an operator on $L^{2}(\mathbb{R})$ if $\sigma$ is a homogeneous distribution on $\mathbb{R}^{2}$.
\end{abstract}

Keywords Weyl transform · Pseudo-differential operators · Homogeneous distributions

Mathematics Subject Classification $47 \mathrm{G} 30 \cdot 46 \mathrm{~F} 10$

\section{Introduction and notation}

In our first paper on Weyl transforms, see [8], we endeavored to generalize the theorems and examples in [11] concerning the boundedness and compactness, respectively, of Weyl transforms of radially symmetric functions to the case of radially symmetric distributions. (Incidentally, let me indicate three printing errors in [8]: On p. 769, the space $\mathcal{D}_{L^{1}}^{\prime}\left(\mathbb{R}^{2}\right)$ should be replaced by $\mathcal{D}_{L^{1}}^{\prime}\left(\mathbb{R}^{1}\right)$ and on p. 775 , the expression $\mathrm{e}^{\mathrm{i} \exp (2 x)}$ was twice contorted to $\mathrm{e}^{\mathrm{i}} \exp (2 x)$; finally, in line 13 on $\mathrm{p} .785$, the factor $s_{0}^{z}$ got lost in the expression $(2 \pi)^{-1} s_{0}^{z} \mathrm{e}^{\mathrm{i} h\left(s_{0}\right)} \sqrt{2 \pi \mathrm{i} / h^{\prime \prime}\left(s_{0}\right)}$.)

In [8], we did not touch on Theorem 27.3 on tensor products in polar coordinates in [11, p. 128] for a variety of reasons: First, this theorem concerns a much more general situation (of which radially symmetric functions are just a tiny special case), second, the setting is much more intricate and arduous than for radially symmetric functions, third, we did not immediately succeed in finding a generalization to distributions with meaningful and manageable examples, and fourth, there is no hope (as far as we can see) for a single necessary and sufficient criterion for boundedness, in contrast to the radial case.

\footnotetext{
$\triangle$ Peter Wagner

wagner@mat1.uibk.ac.at

1 University of Innsbruck, Technikerstr. 13, 6020 Innsbruck, Austria
}

Birkhäuser 
This paper tries to work out [11, Thm. 27.3, p. 128] from a distribution-theoretic viewpoint. We give a distributional formulation of it in Proposition 1, and we specialize Proposition 1 to the case of homogeneous distributions $\sigma$ on $\mathbb{R}^{2}$ in Proposition 4. Therein we state a sufficient condition for the boundedness of the Weyl transform $W_{\sigma}$ on $L^{2}(\mathbb{R})$ in terms of the Fourier coefficients of the characteristic $F$ of $\sigma$. In the preparation for the general case, we deal with the case of homogeneity 0 in Proposition 2, where the matrix coefficients of the Weyl transforms can be expressed more explicitly than in the general case. In Proposition 3, we give necessary conditions on the degree $\lambda$ of homogeneity and on the characteristic $F$ of $\sigma=F \cdot r^{\lambda}$ that must be satisfied in order that $W_{\sigma}$ can be bounded.

Let us introduce some notation. Besides the familiar Banach spaces $L^{p}\left(\mathbb{R}^{n}\right)$, $1 \leq p \leq \infty$, we use the Banach spaces of bounded and of compact linear operators on $\overline{L^{2}}\left(\mathbb{R}^{n}\right)$, respectively, which we denote by $\mathcal{L}\left(L^{2}\left(\mathbb{R}^{n}\right)\right)$ and by $\operatorname{Com}\left(L^{2}\left(\mathbb{R}^{n}\right)\right)$, respectively.

We employ the standard notation for the distribution spaces $\mathcal{D}^{\prime}, \mathcal{S}^{\prime}, \mathcal{E}^{\prime}$, the dual spaces of the spaces $\mathcal{D}, \mathcal{S}, \mathcal{E}$ of "test functions", of "rapidly decreasing functions" and of $C^{\infty}$ functions, respectively, see [4,9]. In order to display the active variable in a distribution, say $x \in \mathbb{R}^{n}$, we use notation as $T(x)$ or $T \in \mathcal{D}^{\prime}\left(\mathbb{R}_{x}^{n}\right)$. For the evaluation of a distribution $T \in E^{\prime}$ on a test function $\phi \in E$, we use angle brackets, i.e., $\langle\phi, T\rangle$ or, more precisely ${ }_{E}\langle\phi, T\rangle_{E^{\prime}}$.

The spaces $\mathcal{D}_{L^{p}}, \mathcal{D}_{L^{p}}^{\prime}, 1 \leq p \leq \infty$, were introduced in [9, Ch. VI, § 8, p. 199], and we need, just for $p=1$ and $p=\infty$, so-called "weighted" $\mathcal{D}_{L^{p}}$ and $\mathcal{D}_{L^{p}}^{\prime}$-spaces, i.e.,

$$
\begin{aligned}
\mathcal{D}_{L^{p}, \mu}:= & \mathcal{D}_{L^{p}} \cdot\left(1+|x|^{2}\right)^{-\mu / 2}, \mathcal{D}_{L^{p}, \mu}^{\prime}:=\mathcal{D}_{L^{p}}^{\prime} \cdot\left(1+|x|^{2}\right)^{-\mu / 2}, \\
& 1 \leq p \leq \infty, \mu \in \mathbb{R},
\end{aligned}
$$

see [6].

$\mathbb{N}, \mathbb{N}_{0}$ and $\mathbb{Z}$ denote the sets of positive, of non-negative, and of all integers, respectively. For sequences with the index sets $\mathbb{N}_{0}$ and $\mathbb{Z}$, respectively, we use the Banach spaces $l^{p}, 1 \leq p \leq \infty$, and the Fréchet space $s$ of fast decreasing sequences with its dual $s^{\prime}$ of slowly increasing sequences.

$\mathbb{S}^{1}$ denotes the unit circle in $\mathbb{R}^{2}$, which we identify with $\mathbb{C}$, and we write $\omega$ for the generic variable on $\mathbb{S}^{1} \subset \mathbb{C}$ and $\mathrm{d} \omega$ for the measure of length on $\mathbb{S}^{1}$. With these notations, $(2 \pi)^{-1 / 2} \omega^{k}, k \in \mathbb{Z}$, is an orthonormal basis in $L^{2}\left(\mathbb{S}^{1}\right)$ and $f(\omega)=\sum_{k \in \mathbb{Z}} c_{k} \omega^{k}$ is the usual Fourier series expansion for $f \in L^{2}\left(\mathbb{S}^{1}\right)$ if $c_{k}=\frac{1}{2 \pi} \int_{\mathbb{S}^{1}} f(\omega) \omega^{-k} \mathrm{~d} \omega$, $k \in \mathbb{Z}$. Furthermore, $L^{1}\left(\mathbb{S}^{1}\right)$ is embedded into $\mathcal{D}^{\prime}\left(\mathbb{S}^{1}\right)$ by $\langle\phi, f\rangle=\int \phi(\omega) f(\omega) \mathrm{d} \omega$.

The Heaviside function is denoted by $Y$, see [9, p. 36], and we write $\delta_{\tau}(t) \in$ $\mathcal{D}^{\prime}\left(\mathbb{R}_{t}^{1}\right), \tau \in \mathbb{R}$, for the delta distribution with support in $\tau$, which is the derivative of $Y(t-\tau)$, i.e., $\left\langle\phi, \delta_{\tau}\right\rangle=\phi(\tau)$ for $\phi \in \mathcal{D}\left(\mathbb{R}^{1}\right)$. In contrast, $\delta \in \mathcal{D}^{\prime}\left(\mathbb{R}^{n}\right)$ without any subscript stands for the delta distribution at the origin. For $z \in \mathbb{C}$ with $\operatorname{Re} z>-1$, we write $t_{+}^{z}$ for the locally integrable function $Y(t) t^{z}$ and we obtain, by analytic continuation, the analytic distribution-valued function

$$
t_{+}^{z}: \mathbb{C} \backslash(-\mathbb{N}) \longrightarrow \mathcal{S}^{\prime}\left(\mathbb{R}_{t}^{1}\right): z \longmapsto t_{+}^{z},
$$


see [7, Ex. 1.4.8, p. 49].

The pull-back $h^{*} T=T \circ h \in \mathcal{D}^{\prime}(\Omega)$ of a distribution $T$ in one variable $t$ with respect to a submersive $\mathcal{C}^{\infty}$ function $h: \Omega \rightarrow \mathbb{R}, \Omega \subset \mathbb{R}^{n}$ open, is defined as in [4, Equ. (7.2.4/5), p. 82] or in [7, Def. 1.2.12, p. 19], i.e.,

$$
\left\langle\phi, h^{*} T\right\rangle=\left\langle\frac{\mathrm{d}}{\mathrm{d} t}\left(\int_{\Omega} Y(t-h(x)) \phi(x) \mathrm{d} x\right), T\right\rangle, \quad \phi \in \mathcal{D}(\Omega) .
$$

We use the Fourier transform $\mathcal{F}$ in the form

$$
(\mathcal{F} \phi)(\xi):=\int_{\mathbb{R}^{n}} \mathrm{e}^{-\mathrm{i} \xi x} \phi(x) \mathrm{d} x, \quad \phi \in \S\left(\mathbb{R}^{n}\right),
$$

this being extended to $\S^{\prime}$ by continuity. For the partial Fourier transforms of a distribution $T \in \mathcal{S}^{\prime}\left(\mathbb{R}_{x y}^{m+n}\right)$ with respect to $x \in \mathbb{R}^{m}$ or $y \in \mathbb{R}^{n}$, respectively, we use the notation $\mathcal{F}_{x} T$ and $\mathcal{F}_{y} T$, respectively.

The Hermite polynomials $H_{j}$ and Hermite functions $h_{j}$ are defined as usually:

$$
H_{j}(x)=(-1)^{j} \mathrm{e}^{x^{2}} \cdot\left(\mathrm{e}^{-x^{2}}\right)^{(j)}, \quad h_{j}(x)=\mathrm{e}^{-x^{2} / 2} H_{j}(x), \quad j \in \mathbb{N}_{0}, x \in \mathbb{R},
$$

see [5, Section 8.95], [11, Sections 18, 19]. Similarly, the Laguerre polynomials $L_{j}^{\alpha}(x)$ of order $\alpha$ are defined by

$$
L_{j}^{\alpha}(x):=j !^{-1} x^{-\alpha} \mathrm{e}^{x} \cdot\left(\mathrm{e}^{-x} x^{\alpha+j}\right)^{(j)}, \quad x>0, \alpha>-1, j \in \mathbb{N}_{0},
$$

see [5, Section 8.97], [11, Section 20, p. 95].

\section{Review of definitions and classical results}

The Weyl transformation $W$ can be described as a partial Fourier transform composed with a linear transformation in $\mathbb{R}_{x y}^{2 n}$, to wit

$$
W: \mathcal{S}^{\prime}\left(\mathbb{R}^{2 n}\right) \longrightarrow \mathcal{S}^{\prime}\left(\mathbb{R}^{2 n}\right): \sigma(x, y) \longmapsto W_{\sigma}(x, y)
$$

where

$$
W_{\sigma}(x, y)=(2 \pi)^{-n}\left(\mathcal{F}_{y} \sigma\right)\left(\frac{x+y}{2}, y-x\right),
$$

see [3, p. 79], [11, Equ. (4.2), p. 19], [8, Sect. 2]. Hence if $\sigma \in \mathcal{S}^{\prime}\left(\mathbb{R}^{2 n}\right)$, then its Weyl transform $W_{\sigma}$ is the pseudo-differential operator represented by the continuous linear mapping $W_{\sigma}: \mathcal{S}\left(\mathbb{R}^{n}\right) \rightarrow \mathcal{S}^{\prime}\left(\mathbb{R}^{n}\right)$ with the kernel $W_{\sigma}(x, y) \in \mathcal{S}^{\prime}\left(\mathbb{R}^{2 n}\right)$, i.e.,

$$
\left(W_{\sigma} \phi\right)(x)=\left\langle\phi(y), W_{\sigma}(x, y)\right\rangle \in \mathcal{S}^{\prime}\left(\mathbb{R}_{x}^{n}\right), \quad \phi \in \mathcal{S}\left(\mathbb{R}^{n}\right) .
$$


The Weyl transform $W$ can be expressed by the "Wigner transform," which is a continuous sesquilinear mapping

$$
w: \mathcal{S}\left(\mathbb{R}^{n}\right) \times \mathcal{S}\left(\mathbb{R}^{n}\right) \longrightarrow \mathcal{S}\left(\mathbb{R}^{2 n}\right) .
$$

The mappings $w$ and $W$ are connected by

$$
\left\langle\bar{\psi}, W_{\sigma} \phi\right\rangle=\langle w(\phi, \psi), \sigma\rangle, \quad \phi, \psi \in \mathcal{S}\left(\mathbb{R}^{n}\right)
$$

This implies

$$
\begin{aligned}
\left\langle\bar{\psi}, W_{\sigma} \phi\right\rangle & =(2 \pi)^{-n}\left\langle\overline{\psi(x)} \phi(y),\left(\mathcal{F}_{y} \sigma\right)\left(\frac{x+y}{2}, y-x\right)\right\rangle \\
& =(2 \pi)^{-n}\left\langle\overline{\psi\left(x-\frac{y}{2}\right)} \phi\left(x+\frac{y}{2}\right), \mathcal{F}_{y} \sigma\right\rangle \\
& =(2 \pi)^{-n}\left\langle\mathcal{F}_{y}\left(\overline{\psi\left(x-\frac{y}{2}\right)} \phi\left(x+\frac{y}{2}\right)\right), \sigma\right\rangle=\langle w(\phi, \psi), \sigma\rangle
\end{aligned}
$$

and hence

$$
(w(\phi, \psi))(x, y)=(2 \pi)^{-n} \int_{\mathbb{R}^{n}} \mathrm{e}^{-\mathrm{i} y \eta} \phi\left(x+\frac{\eta}{2}\right) \overline{\psi\left(x-\frac{\eta}{2}\right)} \mathrm{d} \eta .
$$

Note that we use for $w$ a normalization constant different from that in [11, Equ. (3.12), p. 15], and that we also changed, for clarity, the notation for the Wigner transform from $W(\phi, \psi)$ to $w(\phi, \psi)$.

In this study, we shall focus on symbols $\sigma$ which are homogeneous distributions in two dimensions, and we shall derive necessary and sufficient criteria, respectively, for the boundedness of the corresponding pseudo-differential $W_{\sigma}$ on $L^{2}\left(\mathbb{R}^{1}\right)$, i.e., criteria on whether $W_{\sigma}$ can be extended to a bounded mapping $L^{2}(\mathbb{R}) \rightarrow L^{2}(\mathbb{R})$, or, in other words, whether the norm $\left\|W_{\sigma} \phi\right\|_{2}$ can be bounded by a constant multiple of $\|\phi\|_{2}$ for $\phi \in \mathcal{S}\left(\mathbb{R}^{1}\right)$.

Let us now recapitulate some basic facts about homogeneous distributions $\sigma$ on $\mathbb{R}^{2}$, see [6,7] for more details. If $\sigma \in \mathcal{D}^{\prime}\left(\mathbb{R}^{2} \backslash\{0\}\right)$ is homogeneous of degree $\lambda \in \mathbb{C}$, then the structure theorem (see [6, Sect. 2.5]) implies that $\sigma$ can be extended to $\mathbb{R}^{2}$ and can be represented in the form $\sigma=F \cdot r^{\lambda}$ where the so-called "characteristic" $F$ belongs to $\mathcal{D}^{\prime}\left(\mathbb{S}^{1}\right)$ and

$$
\left.\left\langle\phi, F \cdot r^{\lambda}\right\rangle={\mathcal{S}\left(\mathbb{R}^{1}\right)}_{\mathcal{D}\left(\mathbb{S}^{1}\right)}\langle\phi(t \omega), F(\omega)\rangle_{\mathcal{D}^{\prime}\left(\mathbb{S}^{1}\right)}, t_{+}^{\lambda+1}\right\rangle_{\mathcal{S}^{\prime}\left(\mathbb{R}^{1}\right)}, \quad \phi \in \mathcal{S}\left(\mathbb{R}^{2}\right)
$$

If we identify $\mathbb{S}^{1} \subset \mathbb{R}^{2} \simeq \mathbb{C}$, then

$$
\mathcal{D}^{\prime}\left(\mathbb{S}^{1}\right) \stackrel{\sim}{\longrightarrow} s^{\prime}(\mathbb{Z}): F \longmapsto\left(c_{k}\right)_{k \in \mathbb{Z}}
$$

where $c_{k}=\frac{1}{2 \pi}\left\langle\omega^{-k}, F\right\rangle \in \mathbb{C}$ for $k \in \mathbb{Z}$ and $\sum_{k \in \mathbb{Z}} c_{k} \omega^{k}$ converges to $F(\omega)$ in $\mathcal{D}^{\prime}\left(\mathbb{S}^{1}\right)$. The Hilbert spaces 


$$
\mathcal{H}^{(\mu)}\left(\mathbb{S}^{1}\right)=\left\{F(\omega)=\sum_{k \in \mathbb{Z}} c_{k} \omega^{k} \in \mathcal{D}^{\prime}\left(\mathbb{S}^{1}\right) ;\left(c_{k}(1+|k|)^{\mu}\right)_{k \in \mathbb{Z}} \in l^{2}(\mathbb{Z})\right\}, \quad \mu \in \mathbb{R},
$$

are the usual Sobolev spaces on $\mathbb{S}^{1}$.

The distribution-valued function $z \mapsto t_{+}^{z}$ is meromorphic with simple poles at $z=-k, k \in \mathbb{N}$. We have $\operatorname{Res}_{z=-k} t_{+}^{z}=(-1)^{k-1}(k-1) !^{-1} \delta^{(k-1)}$ and we set

$$
t_{+}^{-k}:=\operatorname{Pf}_{z=-k} t_{+}^{z}=\lim _{z \rightarrow-k}\left(t^{z}-(z+k)^{-1} \operatorname{Res}_{\lambda=-k} t^{\lambda}\right), \quad k \in \mathbb{N} .
$$

For $\lambda \in \mathbb{C} \backslash\left(-2-\mathbb{N}_{0}\right)$, this implies that $F \cdot r^{\lambda}$ is homogeneous not only in $\mathbb{R}^{2} \backslash\{0\}$, but also on $\mathbb{R}^{2}$, whereas for $\lambda=-2-j, j \in \mathbb{N}_{0}, F \cdot r^{\lambda}$ is homogeneous on $\mathbb{R}^{2}$ if and only if the Fourier coefficients $c_{k}=(2 \pi)^{-1}\left\langle\omega^{-k}, F\right\rangle$ vanish for $|k| \leq j, k \in \mathbb{Z}$, see [6, Thm. 2.5.1, p. 58].

Furthermore, if $\mathcal{S}_{\lambda}^{\prime}\left(\mathbb{R}^{2}\right), \lambda \in \mathbb{C}$, denotes the closed subspace of $\mathcal{S}^{\prime}\left(\mathbb{R}^{2}\right)$ of distributions which are homogeneous of degree $\lambda$ in $\mathbb{R}^{2}$, then $\mathcal{F}: \mathcal{S}_{\lambda}^{\prime}\left(\mathbb{R}^{2}\right) \stackrel{\sim}{\rightarrow} \mathcal{S}_{-2-\lambda}^{\prime}\left(\mathbb{R}^{2}\right)$ is an isomorphism of locally convex spaces. More explicitly, if $\lambda \in \mathbb{C} \backslash \mathbb{Z}$, then $\mathcal{F}\left(F \cdot r^{\lambda}\right)=G \cdot r^{-2-\lambda}$ where $F=\sum_{k \in \mathbb{Z}} c_{k} \omega^{k}, G=\sum_{k \in \mathbb{Z}} d_{k} \omega^{k}$ and

$$
d_{k}=2^{2+\lambda} \pi \mathrm{i}^{k} \frac{\Gamma\left(1+\frac{\lambda-k}{2}\right)}{\Gamma\left(-\frac{\lambda+k}{2}\right)} c_{k}, \quad k \in \mathbb{Z}
$$

Formula (2.2) is a special case of the Bochner-Hecke formula, see [10, Ch. IV, Thm. 4.1, p. 160]. For a direct verification, note that, with $z=x+\mathrm{i} y=r \omega, r=|z|$, and using [7, Equ. (1.6.18), p. 99], we have

$$
\begin{aligned}
\mathcal{F}\left(\omega^{k} \cdot r^{\lambda}\right) & =\mathcal{F}\left(z^{k} \cdot r^{\lambda-k}\right)=\mathrm{i}^{|k|}\left(\partial_{x}+\operatorname{sign}(k) \mathrm{i} \partial_{y}\right)^{|k|} \mathcal{F}\left(r^{\lambda-|k|}\right) \\
& =2^{2+\lambda} \pi \mathrm{i}^{|k|} \Gamma\left(1+\frac{\lambda-|k|}{2}\right)\left(\frac{1}{2}\left(\partial_{x}+\operatorname{sign}(k) \mathrm{i} \partial_{y}\right)\right)^{|k|}\left(\frac{r^{|k|-2-\lambda}}{\Gamma\left(\frac{|k|-\lambda}{2}\right)}\right) \\
& =2^{2+\lambda} \pi \mathrm{i}^{|k|} \frac{\Gamma\left(1+\frac{\lambda-|k|}{2}\right)}{\Gamma\left(-\frac{\lambda+|k|}{2}\right)} \omega^{k} \cdot r^{-2-\lambda}, \quad k \in \mathbb{Z} .
\end{aligned}
$$

We finally observe that the factor in front of $c_{k}$ in formula (2.2) does not depend on the sign of $k$ on account of the complement formula for the gamma function.

As we shall see in Proposition 3 below, a homogeneous symbol $\sigma \in \mathcal{S}^{\prime}\left(\mathbb{R}^{2}\right)$ can have a bounded Weyl transform $W_{\sigma}$ only under the condition that the degree $\lambda$ of homogeneity fulfills $-2 \leq \operatorname{Re} \lambda \leq 0$. For homogeneous and radially symmetric symbols $\sigma=r^{\lambda} \in \mathcal{S}^{\prime}\left(\mathbb{R}^{2}\right)$, we have shown in [8, Sect. 5, Ex. 1] that $W_{\sigma}$ is bounded on $L^{2}\left(\mathbb{R}^{1}\right)$ if and only if $-2 \leq \operatorname{Re} \lambda \leq 0, \lambda \neq-2$. (Note that $r^{-2}=\operatorname{Pf}_{\lambda=-2} r^{\lambda}$ is not homogeneous on $\mathbb{R}^{2}$. We remark, incidentally, that $W_{\sigma}$ is unbounded for $\sigma=r^{-2} \in$ $\mathcal{S}^{\prime}\left(\mathbb{R}^{2}\right)$, see the remark to Proposition 3.)

Let us describe next what can be inferred from classical results. According to [11, Thms. 11.1, 11.3, pp. 55, 57], compare also [8, Prop. 1, p. 773], $W_{\sigma}$ is bounded and even compact as an operator on $L^{2}\left(\mathbb{R}^{n}\right)$ if $\sigma$ or $\mathcal{F} \sigma$ belong to $\cup_{p \in[1,2]} L^{p}\left(\mathbb{R}^{2 n}\right)$. In particular, 
if $\sigma=F(\omega) \cdot r^{\lambda} \in \mathcal{S}^{\prime}\left(\mathbb{R}^{2}\right)$ with $F \in L^{2}\left(\mathbb{S}^{1}\right)$ and $-2<\operatorname{Re} \lambda<-1$, then $\sigma \in$ $L^{1}\left(\mathbb{R}^{2}\right)+L^{2}\left(\mathbb{R}^{2}\right)$ and hence $W_{\sigma} \in \operatorname{Com}\left(L^{2}\left(\mathbb{R}^{1}\right)\right)$. Similarly, if $-1<\mu=\operatorname{Re} \lambda<0$, and $F \in \mathcal{H}^{(\mu+1)}\left(\mathbb{S}^{1}\right)$, i.e., $F(\omega)=\sum_{k \in \mathbb{Z}} c_{k} \omega^{k}$ such that $\left(c_{k}|k|^{\mu+1}\right)_{k \in \mathbb{Z}} \in l^{2}(\mathbb{Z})$, then we employ formula (2.2) and conclude once again that $W_{\sigma} \in \operatorname{Com}\left(L^{2}\left(\mathbb{R}^{1}\right)\right)$ from the asymptotic behaviour

$$
\frac{\Gamma\left(1+\frac{\lambda-|k|}{2}\right)}{\Gamma\left(-\frac{\lambda+|k|}{2}\right)}=(-1)^{k} \frac{\Gamma\left(1+\frac{|k|+\lambda}{2}\right)}{\Gamma\left(\frac{|k|-\lambda}{2}\right)} \sim(-1)^{k}\left(\frac{|k|}{2}\right)^{\lambda+1}, \quad|k| \rightarrow \infty,
$$

see [5, Equ. 8.328.2]. We shall deduce more general results in Sect. 6 below.

\section{The matrix representation of $w_{\sigma}$}

The normalized Hermite functions

$$
e_{j}=h_{j} /\left\|h_{j}\right\|_{2}=\left(2^{j} j ! \sqrt{\pi}\right)^{-1 / 2} h_{j} \in \mathcal{S}(\mathbb{R}), \quad j \in \mathbb{N}_{0},
$$

are an orthonormal basis in $L^{2}(\mathbb{R})$, see, e.g., [11, Thm. 19.3, p. 94]. This yields an isomorphism of Hilbert spaces:

$$
L^{2}(\mathbb{R}) \stackrel{\sim}{\longrightarrow} l^{2}\left(\mathbb{N}_{0}\right): f \longmapsto\left(\int_{\mathbb{R}} f(x) e_{j}(x) \mathrm{d} x\right)_{j \in \mathbb{N}_{0}} .
$$

As was observed already by Schwartz, cf. [9, Ch. VII, Sect. 7, Ex. 7, pp. 260, 262], the sequence $e_{j}, j \in \mathbb{N}_{0}$, also serves to identify the spaces $\mathcal{S}(\mathbb{R})$ and $\mathcal{S}^{\prime}(\mathbb{R})$ with the sequence spaces $s\left(\mathbb{N}_{0}\right)$ and $s^{\prime}\left(\mathbb{N}_{0}\right)$, respectively. Hence we obtain a commutative diagram:

$$
\begin{array}{ccccc}
\mathcal{S}(\mathbb{R}) & \hookrightarrow & L^{2}(\mathbb{R}) & \hookrightarrow & \mathcal{S}^{\prime}(\mathbb{R}) \\
\downarrow^{2} & & \downarrow^{2} & & \downarrow^{2} \\
s\left(\mathbb{N}_{0}\right) & \hookrightarrow & l^{2}\left(\mathbb{N}_{0}\right) & \hookrightarrow & s^{\prime}\left(\mathbb{N}_{0}\right)
\end{array}
$$

Here the vertical isomorphisms in the diagram are all given by $T \mapsto\left(\left\langle e_{j}, T\right\rangle\right)_{j \in \mathbb{N}_{0}}$.

By the same token, using $\mathcal{S}^{\prime}\left(\mathbb{R}^{2}\right) \stackrel{\sim}{\rightarrow} s^{\prime}\left(\mathbb{N}_{0}^{2}\right)$, the mapping $W_{\sigma}: \mathcal{S}(\mathbb{R}) \rightarrow \mathcal{S}^{\prime}(\mathbb{R})$ is represented by the matrix $B_{\sigma}=\left(b_{j k}\right)_{j, k \in \mathbb{N}_{0}} \in s^{\prime}\left(\mathbb{N}_{0}^{2}\right)$ where $W_{\sigma} e_{k}=\sum_{j \in \mathbb{N}_{0}} e_{j} b_{j k}$ for $k \in \mathbb{N}_{0}$ and the sums converge in $\mathcal{S}^{\prime}(\mathbb{R})$. The matrix coefficients $b_{j k}$ of $W_{\sigma}$ are given by

$$
b_{j k}=\left\langle e_{j}, W_{\sigma} e_{k}\right\rangle=\left\langle w\left(e_{k}, e_{j}\right), \sigma\right\rangle, \quad j, k \in \mathbb{N}_{0},
$$

on account of formula (2.1). Therefore, the operator $W_{\sigma}$ is bounded on $L^{2}(\mathbb{R})$ if and only if

$$
\exists C>0: \forall \phi \in s\left(\mathbb{N}_{0}\right):\left\|\left(\sum_{k=0}^{\infty} b_{j k} \phi_{k}\right)_{j \in \mathbb{N}_{0}}\right\|_{l^{2}} \leq C\|\phi\|_{l^{2}}
$$

holds. 
Let us specialize now to distributions $\sigma \in \mathcal{S}^{\prime}\left(\mathbb{R}^{2}\right)$ that are tensor products in polar coordinates. For the radial dependency of $\sigma$, we take $R \in \mathcal{S}^{\prime}([0, \infty))$, which is the dual space of $\mathcal{S}([0, \infty))=\left\{\left.\phi\right|_{[0, \infty)}: \phi \in \mathcal{S}(\mathbb{R})\right\}$ equipped with the seminorms

$$
\|\phi\|_{k, l}=\left\|(1+x)^{k} \phi^{(l)}(x)\right\|_{L^{\infty}([0, \infty))}, \quad k, l \in \mathbb{N}_{0} .
$$

As in $[8$, p. 776], we observe that

$$
\mathcal{S}^{\prime}([0, \infty)) \stackrel{\sim}{\longrightarrow}\left\{S \in \mathcal{S}^{\prime}(\mathbb{R}) ; \operatorname{supp} S \subset[0, \infty)\right\}: S \longmapsto\left(\phi \mapsto\left\langle\left.\phi\right|_{[0, \infty)}, S\right\rangle\right)
$$

is an isomorphism of locally convex spaces.

If, furthermore, $F \in \mathcal{D}^{\prime}\left(\mathbb{S}^{1}\right)$, then we define $F \cdot R \in \mathcal{S}^{\prime}\left(\mathbb{R}^{2}\right)$ by the following:

$$
\langle\phi, F \cdot R\rangle=\mathcal{S}_{([0, \infty))}\langle r \cdot\langle\phi(r \omega), F(\omega)\rangle, R(r)\rangle_{\mathcal{S}^{\prime}([0, \infty))}, \quad \phi \in \mathcal{S}\left(\mathbb{R}^{2}\right) .
$$

Note that this is well-defined since the function

$$
\mathbb{R} \longrightarrow \mathbb{C}: r \longmapsto r \cdot\langle\phi(r \omega), F(\omega)\rangle
$$

belongs to $\mathcal{S}\left(\mathbb{R}^{1}\right)$, but that the distribution $R \in \mathcal{S}^{\prime}([0, \infty))$ is not uniquely determined up to a factor by the distribution $\sigma=F \cdot R$ since, e.g., $F \cdot \delta=0$.

In the following Proposition 1 , we state formulas for the matrix coefficients of $W_{\sigma}$ with respect to the normalized Hermite functions $e_{j}, e_{k}$ if $\sigma=F \cdot R \in \mathcal{S}^{\prime}\left(\mathbb{R}^{2}\right)$ is a tensor product in polar coordinates. This proposition is but a slight extension of Theorem 27.3 in [11, p. 128] from temperate functions to temperate distributions.

Proposition 1 Let $e_{j}$ be the normalized Hermite functions as in formula (3.1) and $L_{k}^{\alpha}$ be the Laguerre polynomials as in Sect. 1. Let $F(\omega)=\sum_{k \in \mathbb{Z}} c_{k} \omega^{k} \in \mathcal{D}^{\prime}\left(\mathbb{S}^{1}\right)$ and $R \in \mathcal{S}^{\prime}\left([0, \infty)\right.$ ) and let $\sigma=F \cdot R \in \mathcal{S}^{\prime}\left(\mathbb{R}^{2}\right)$ be defined as in formula (3.3). Let $W_{\sigma} e_{k}=\sum_{j \in \mathbb{N}_{0}} e_{j} b_{j k}, k \in \mathbb{N}_{0}$, with $\left(b_{j k}\right)_{j, k \in \mathbb{N}_{0}} \in s^{\prime}\left(\mathbb{N}_{0}^{2}\right)$ being the matrix representation of $W_{\sigma}$ and define the symmetric matrix $\left(a_{j k}\right)_{j, k \in \mathbb{N}_{0}} \in s^{\prime}\left(\mathbb{N}_{0}^{2}\right)$ by

$$
a_{j k}=2(-1)^{k} \sqrt{\frac{k !}{j !}} 2^{(j-k) / 2} \mathcal{S}([0, \infty))\left\langle r^{j-k+1} L_{k}^{j-k}\left(2 r^{2}\right) \mathrm{e}^{-r^{2}}, R(r)\right\rangle_{\mathcal{S}^{\prime}([0, \infty))}, j \geq k
$$

and $a_{j k}=a_{k j}$ for $j \leq k, j, k \in \mathbb{N}_{0}$.

Then the following holds:

(a) For $j, k \in \mathbb{N}_{0}$ we have $b_{j k}=a_{j k} c_{k-j}$;

(b) if $\mu \in \mathbb{R}$ and

$$
\exists C>0: \forall j, k \in \mathbb{N}_{0}:\left|a_{j k}\right| \leq C \cdot(1+|j-k|)^{\mu}
$$

and $\left((1+|k|)^{\mu} c_{k}\right)_{k \in \mathbb{Z}} \in l^{1}(\mathbb{Z})$, then $W_{\sigma}$ is bounded, i.e., $W_{\sigma} \in \mathcal{L}\left(L^{2}(\mathbb{R})\right)$. 
Proof (a) The formula for $b_{j k}$ in the proposition directly follows from the calculation of the Wigner transforms $w\left(e_{j}, e_{k}\right), j, k \in \mathbb{N}_{0}$. This calculation goes back to $C$. Itzykson, W. Miller, and N.Ya. Vilenkin, see the historical comment in [3, p. 65]. Taking account of the different notations in the literature, we have, for $j, k \in \mathbb{N}_{0}$ with $j \geq k$,

$$
\left(w\left(e_{j}, e_{k}\right)\right)(r \omega)=\frac{2(-1)^{k}}{2 \pi} \sqrt{\frac{k !}{j !}} 2^{(j-k) / 2} r^{j-k} \omega^{k-j} L_{k}^{j-k}\left(2 r^{2}\right) \mathrm{e}^{-r^{2}}
$$

where $r>0$ and $\omega \in \mathbb{S}^{1}$, see [11, Thm. 24.1, p. 113], [3, Thm. 1.104, p. 64]. Furthermore we have, quite trivially, $w\left(e_{k}, e_{j}\right)=\overline{w\left(e_{j}, e_{k}\right)}$ for $j, k \in \mathbb{N}_{0}$.

Hence employing formulas (3.2), (3.3), (3.4) and (3.6), we obtain

$$
b_{j k}=\left\langle w\left(e_{k}, e_{j}\right), \sigma\right\rangle=\left\langle r \cdot\left\langle\overline{w\left(e_{j}, e_{k}\right)(r \omega)}, F(\omega)\right\rangle, R(r)\right\rangle=a_{j k} c_{k-j}, \quad j, k \in \mathbb{N}_{0} .
$$

(b) In the second part, we will closely follow the proof of Theorem 27.3 in [11, p. 129]. Assuming condition (3.5) and $\left((1+|k|)^{\mu} c_{k}\right)_{k \in \mathbb{Z}} \in l^{1}(\mathbb{Z})$, we set $d_{k}=C(1+|k|)^{\mu}\left|c_{k}\right|$, $k \in \mathbb{Z}$. Then the sequence $\left(d_{k}\right)_{k \in \mathbb{Z}}$ belongs to $l^{1}(\mathbb{Z})$ and

$$
\left|b_{j k}\right|=\left|a_{j k}\right| \cdot\left|c_{k-j}\right| \leq C \cdot(1+|k-j|)^{\mu}\left|c_{k-j}\right|=d_{k-j}, \quad j, k \in \mathbb{N}_{0} .
$$

Hence, if $\phi \in s\left(\mathbb{N}_{0}\right)$ and if we set $\phi_{k}=0$ for $k<0$, then the matrix $B_{\sigma}=\left(b_{j k}\right)_{j, k \in \mathbb{N}_{0}}$ of $W_{\sigma}$ fulfills

$$
\begin{aligned}
\left\|B_{\sigma} \phi\right\|_{l^{2}\left(\mathbb{N}_{0}\right)} & =\left\|\left(\sum_{k=0}^{\infty} b_{j k} \phi_{k}\right)_{j \in \mathbb{N}_{0}}\right\|_{l^{2}\left(\mathbb{N}_{0}\right)} \leq\left\|\left(\sum_{k \in \mathbb{Z}} d_{k-j}\left|\phi_{k}\right|\right)_{j \in \mathbb{N}_{0}}\right\|_{l^{2}\left(\mathbb{N}_{0}\right)} \\
& =\left\|\left(\sum_{l \in \mathbb{Z}} d_{l}\left|\phi_{j+l}\right|\right)_{j \in \mathbb{N}_{0}}\right\|_{l^{2}\left(\mathbb{N}_{0}\right)} \leq\|\phi\|_{l^{2}\left(\mathbb{N}_{0}\right)} \sum_{l \in \mathbb{Z}} d_{l},
\end{aligned}
$$

i.e., $B_{\sigma}$ and thus also $W_{\sigma}$ are bounded. This completes the proof.

Remarks (1) Let us first discuss the special case of $\sigma \in \mathcal{S}^{\prime}\left(\mathbb{R}^{2}\right)$ being radially symmetric. We can set then $F=1$ and hence $\sigma$ is given by $R(r) \in \mathcal{S}^{\prime}([0, \infty))$. As we have mentioned above, $R$ is not uniquely determined by $\sigma$. However, as explained in [8, p. 776], there is a unique $S \in \mathcal{S}^{\prime}([0, \infty))$ such that $\sigma$ is represented by the formula

$$
\langle\phi, \sigma\rangle=\mathcal{S}([0, \infty))\left\langle\frac{1}{2} \int_{\mathbb{S}^{1}} \phi(\sqrt{t} \omega) \mathrm{d} \omega, S(t)\right\rangle_{\mathcal{S}^{\prime}([0, \infty))}, \quad \phi \in \mathcal{S}\left(\mathbb{R}^{2}\right)
$$

This means that $S \in \mathcal{S}^{\prime}([0, \infty))$ satisfies

$$
\left\langle 2 r \psi\left(r^{2}\right), R(r)\right\rangle=\langle\psi, S\rangle, \quad \psi \in \mathcal{S}([0, \infty)) .
$$


If we set $F=1$ in Proposition 1 , we obtain $c_{k}=\delta_{k 0}$ and hence the matrix $B_{\sigma}$ is diagonal with the eigenvalues

$$
\lambda_{j}=a_{j j}=2(-1)^{j}\left\langle r L_{j}^{0}\left(2 r^{2}\right) \mathrm{e}^{-r^{2}}, R(r)\right\rangle=(-1)^{j}\left\langle L_{j}^{0}(2 t) \mathrm{e}^{-t}, S(t)\right\rangle, \quad j \in \mathbb{N}_{0}
$$

Formula (3.8) coincides with [8, Prop. 3, Equ. (3.5), p. 777].

However note that condition (3.5) in Proposition 1 above is overly restrictive in the case of radially symmetric distributions since, clearly, the diagonal matrix $B_{\sigma}$ is bounded on $l^{2}\left(\mathbb{N}_{0}\right)$ if and only if the sequence of eigenvalues $\left(a_{j j}\right)_{j \in \mathbb{N}_{0}}$ is bounded. Hence no restriction on $a_{j k}, j \neq k$, is necessary. More generally, if $F$ is a trigonometric polynomial, i.e., if $F(\omega)=\sum_{k \in M} c_{k} \omega^{k}$ where $M$ is a finite subset of $\mathbb{Z}$ with $c_{k} \neq 0$ for $k \in M$ and if $\sigma=F \cdot R$, then $W_{\sigma}$ is bounded on $L^{2}(\mathbb{R})$ if and only if the set $\left\{a_{j k} ; k-j \in M\right\}$ is bounded in $\mathbb{C}$.

(2) In [8, Prop. 4, Equ. (4.2), p. 779], a representation of the eigenvalues $\lambda_{j}=a_{j j}$, $j \in \mathbb{N}_{0}$, by integrals with rational kernels was derived for $\sigma \in \mathcal{S}^{\prime}\left(\mathbb{R}^{2}\right)$ radially symmetric and such that $\sigma(x, y)=(\mathcal{F} T)\left(x^{2}+y^{2}\right)$ with $T \in \mathcal{D}_{L^{1},-1}^{\prime}(\mathbb{R})$. This formula, i.e.,

$$
\lambda_{j}=\mathcal{D}_{L^{\infty}, 1}\left\langle\frac{(1-\mathrm{i} t)^{j}}{(1+\mathrm{i} t)^{j+1}}, T(t)\right\rangle_{\mathcal{D}_{L^{1},-1}^{\prime}}, \quad j \in \mathbb{N}_{0},
$$

was useful in some examples in calculating the asymptotic behavior of $\lambda_{j}$ for $j \rightarrow \infty$, see [8, Sect. 5].

Since we will make no use here of the corresponding "rational" representation of $a_{j k}$, let us just state the formula without proof: If $j, k \in \mathbb{N}_{0}, j \geq k, T \in \mathcal{D}_{L^{1},-1}^{\prime}(\mathbb{R})$ and $R(r)=(\mathcal{F} T)\left(r^{2}\right) \cdot r^{j-k}$ holds in $\mathcal{S}^{\prime}([0, \infty))$, then

$$
a_{j k}=\sqrt{\frac{j !}{k !}} 2^{(j-k) / 2} \mathcal{D}_{L^{\infty}, 1}\left\langle\frac{(1-\mathrm{i} t)^{k}}{(1+\mathrm{i} t)^{j+1}}, T(t)\right\rangle_{\mathcal{D}_{L^{1},-1}^{\prime}} .
$$

As in the diagonal case, formula (3.9) can be derived from formula (3.4) by Fourier transformation, compare the procedure in [11, Sects. 25, 26].

\section{The case of homogeneous symbols of degree 0}

We specialize now our investigation to homogeneous symbols $\sigma=F \cdot r^{\lambda}, \lambda \in \mathbb{C}$, $F(\omega)=\sum_{k \in \mathbb{Z}} c_{k} \omega^{k} \in \mathcal{D}^{\prime}\left(\mathbb{S}^{1}\right)$. We assume that $\sigma$ is homogeneous on $\mathbb{R}^{2}$ and hence that $c_{k}=0$ for $|k| \leq j$ if $\lambda=-2-j, j \in \mathbb{N}_{0}$, cf. Sect. 2 .

In order to calculate $a_{j k}(\lambda)$ for $R(r)=r_{+}^{\lambda} \in \mathcal{S}^{\prime}([0, \infty))$ according to formula (3.4), we first assume that $\lambda \in \mathbb{C} \backslash \mathbb{Z}$ with $\operatorname{Re} \lambda>-2$. Then [1, Equ. 11.44, p. 110] 
and [5, Equ. 9.131.1] yield the following for $j, k \in \mathbb{N}_{0}$ with $j \geq k$ :

$$
\begin{aligned}
a_{j k}(\lambda) & =2(-1)^{k} \sqrt{\frac{k !}{j !}} 2^{(j-k) / 2} \int_{0}^{\infty} r^{\lambda+j-k+1} L_{k}^{j-k}\left(2 r^{2}\right) \mathrm{e}^{-r^{2}} \mathrm{~d} r \\
& =\frac{(-1)^{k}}{2^{\lambda / 2+1}} \sqrt{\frac{k !}{j !}} \int_{0}^{\infty} t^{(\lambda+j-k) / 2} L_{k}^{j-k}(t) \mathrm{e}^{-t / 2} \mathrm{~d} t \\
& =2^{(j-k) / 2} \frac{\Gamma\left(\frac{\lambda+j+k}{2}+1\right)}{\sqrt{j ! k !}} \cdot{ }_{2} F_{1}\left(-k, \frac{j-k-\lambda}{2} ;-\frac{j+k+\lambda}{2} ;-1\right) \\
& =2^{(j+k) / 2} \frac{\Gamma\left(\frac{\lambda+j+k}{2}+1\right)}{\sqrt{j ! k !}} \cdot{ }_{2} F_{1}\left(-j,-k ;-\frac{j+k+\lambda}{2} ; \frac{1}{2}\right)
\end{aligned}
$$

By analytic continuation, formulas (4.1), (4.2) hold for $\lambda \in \mathbb{C} \backslash \mathbb{Z}$ and, due to the symmetry of (4.2) in $j, k$, for all $j, k \in \mathbb{N}_{0}$. But note that the hypergeometric function ${ }_{2} F_{1}(a, b ; c ; z)$ is defined for $c \in-\mathbb{N}_{0}$ only if $\left[a \in-\mathbb{N}_{0}\right.$ and $\left.a>c\right]$ or $\left[b \in-\mathbb{N}_{0}\right.$ and $b>c]$. Because of that and because of the gamma factor in (4.1), (4.2), we have to exercise some care if $\lambda \in \mathbb{Z}$.

Let us now specialize further and consider the case $\lambda=0$, i.e., the case of homogeneous symbols $\sigma=F(\omega)$ of degree 0 .

Proposition 2 Let $F(\omega)=\sum_{k \in \mathbb{Z}} c_{k} \omega^{k} \in \mathcal{D}^{\prime}\left(\mathbb{S}^{1}\right)$ and $\sigma=F \cdot 1 \in \mathcal{S}^{\prime}\left(\mathbb{R}^{2}\right)$ be homogeneous of degree 0 . Then the following holds:

(a) If $\alpha_{j}=\sqrt{\Gamma\left(\frac{j}{2}+1\right) / \Gamma\left(\frac{j+1}{2}\right)}$ for $j \in \mathbb{N}_{0}$ and $a_{j k}=a_{j k}(0)$ is as in Proposition 1 for $R(r)=1$, then

$$
a_{j k}(0)=\left\{\begin{array}{l}
\frac{\alpha_{\max (j, k)}}{\alpha_{\min (j, k)}}: \min (j, k) \text { is even }, \\
\frac{\alpha_{\min (j, k)}}{\alpha_{\max (j, k)}}: \min (j, k) \text { is odd }
\end{array}\right\}, \quad j, k \in \mathbb{N}_{0}
$$

(b) If $\left(\sqrt[4]{|k|} c_{k}\right)_{k \in \mathbb{Z}} \in l^{1}(\mathbb{Z})$, then $W_{\sigma} \in \mathcal{L}\left(L^{2}(\mathbb{R})\right)$.

Proof (a) If $j=k \in \mathbb{N}_{0}$, then formula (4.1) yields $a_{j j}(0)=1$. If $j \neq k$, then formula (4.2) is valid. Let us first assume $j>k$ and $k$ even. For $\operatorname{Re} z<-k$, [2, Equ. 7.3.7.4] yields

$$
{ }_{2} F_{1}\left(z,-k ; \frac{z-k}{2} ; \frac{1}{2}\right)=\sqrt{\pi} \frac{\Gamma\left(\frac{z-k}{2}\right)}{\Gamma\left(\frac{z}{2}\right) \Gamma\left(\frac{1-k}{2}\right)}=\frac{\Gamma\left(1-\frac{z}{2}\right) \Gamma\left(\frac{k+1}{2}\right)}{\sqrt{\pi} \Gamma\left(\frac{k-z}{2}+1\right)}
$$

and hence

$$
a_{j k}(0)=2^{(j+k) / 2} \frac{\Gamma\left(\frac{j}{2}+1\right) \Gamma\left(\frac{k+1}{2}\right)}{\sqrt{\pi \Gamma(j+1) \Gamma(k+1)}}=\frac{\alpha_{j}}{\alpha_{k}}
$$

on account of the complement formula and of the duplication formula for the gamma function. 
Similarly, if $k$ is odd and $\operatorname{Re} z<-k$, then [2, Equ. 7.3.7.4] furnishes

$$
{ }_{2} F_{1}\left(z,-k ; \frac{z-k}{2} ; \frac{1}{2}\right)=\sqrt{\pi} \frac{\Gamma\left(\frac{z-k}{2}\right)}{\Gamma\left(\frac{z+1}{2}\right) \Gamma\left(-\frac{k}{2}\right)}=\frac{\Gamma\left(\frac{1-z}{2}\right) \Gamma\left(\frac{k}{2}+1\right)}{\sqrt{\pi} \Gamma\left(\frac{k-z}{2}+1\right)}
$$

and hence $a_{j k}(0)=\alpha_{k} / \alpha_{j}$ for $j>k$. This proves formula (4.3).

(b) Let us next estimate $a_{j k}(0)$. Using Euler's psi function $\psi(x)=(\log \Gamma(x))^{\prime}$ for $x>0$ we obtain

$$
\log \alpha_{j}=\frac{1}{2} \int_{(j+1) / 2}^{j / 2+1} \psi(x) \mathrm{d} x
$$

Equation (4.4) implies, incidentally, that $\alpha_{j}$ is monotonically increasing since the same holds for the function $\psi$ due to the logarithmic convexity of the gamma function, see, e.g., [5, Equ. 8.363.3].

Since $C_{1}+\log x \leq \psi(x) \leq C_{2}+\log x$ holds for all $x \geq \frac{1}{2}$ and appropriate constants $C_{1}, C_{2} \in \mathbb{R}$, we conclude that

$$
\exists C>0: \forall j \in \mathbb{N}_{0}: C^{-1} \sqrt[4]{j+1} \leq \alpha_{j} \leq C \sqrt[4]{j+1}
$$

This implies

$$
\begin{aligned}
\exists C & >0: \forall j, k \in \mathbb{N}_{0} \text { with } j \geq k: 0 \leq a_{j k}(0) \leq \frac{\alpha_{j}}{\alpha_{k}} \\
& \leq C \sqrt[4]{\frac{j+1}{k+1}}=C\left(1+\frac{j-k}{k+1}\right)^{1 / 4} .
\end{aligned}
$$

Therefore, condition (3.5) in Proposition 1 holds for $\mu=\frac{1}{4}$ and this implies that $W_{\sigma}$ is bounded on $L^{2}(\mathbb{R})$ if the condition $\left(\sqrt[4]{|k|} c_{k}\right)_{k \in \mathbb{Z}} \in l^{1}(\mathbb{Z})$ is assumed. The proof is complete.

Remark The matrix $\left(a_{j k}(\lambda)\right)_{j, k \in \mathbb{N}_{0}}$ can be calculated similarly for each integral $\lambda$. E.g., if $\lambda=-1$, then [2, Equ. 7.3.7.5] implies that $a_{j k}(-1)=\left(\alpha_{j} \alpha_{k}\right)^{-1}$ if $\min (j, k)$ is even and $a_{j k}(-1)$ vanishes if $\min (j, k)$ is odd. Hence

$$
\exists C>0: \forall j, k \in \mathbb{N}_{0}: 0 \leq a_{j k}(-1) \leq C(1+|j-k|)^{-1 / 4}
$$

and $W_{\sigma} \in \mathcal{L}\left(L^{2}(\mathbb{R})\right)$ for $\sigma=F \cdot r^{-1}$ if $\sum_{k \in \mathbb{Z}}\left|c_{k}\right|(1+|k|)^{-1 / 4}<\infty$.

\section{Necessary conditions}

We shall prove here that $\sigma \in \mathcal{S}^{\prime}\left(\mathbb{R}^{2}\right)$ homogeneous of degree $\lambda$ can have a bounded Weyl transform only if the real part of $\lambda$ lies in the interval $[-2,0]$ and if the characteristic $F$ of $\sigma$ belongs to the Sobolev space $\mathcal{H}^{(1 / 4+\mu / 2)}\left(\mathbb{S}^{1}\right)$. In deriving this result, we need yet another representation of $a_{j k}(\lambda)$ by the hypergeometric function. 
If we use the second equation in [1, Equ. 11.44, p. 110] or, alternatively, apply (employing a limit procedure) [5, Equ. 9.131.2] to formula (4.1), then we obtain the following for $\lambda \in \mathbb{C} \backslash \mathbb{Z}$ and $j, k \in \mathbb{N}_{0}$ with $j \geq k$ :

$$
a_{j k}(\lambda)=2^{(j-k) / 2} \sqrt{\frac{j !}{k !}} \frac{\Gamma\left(\frac{\lambda+j-k}{2}+1\right)}{(j-k) !} \cdot{ }_{2} F_{1}\left(-k, \frac{j-k-\lambda}{2} ; j-k+1 ; 2\right) .
$$

Let us now digress a little bit and derive the "complement formula" for the matrix coefficients $a_{j k}(\lambda)$ from formula (5.1). Upon applying [5, Equ. 9.131.1] to formula (5.1) we obtain, still for $j \geq k$,

$$
a_{j k}(\lambda)=2^{(j-k) / 2} \sqrt{\frac{j !}{k !}} \frac{\Gamma\left(\frac{\lambda+j-k}{2}+1\right)}{(j-k) !} \cdot(-1)^{k}{ }_{2} F_{1}\left(-k, \frac{j-k+\lambda+2}{2} ; j-k+1 ; 2\right),
$$

wherefrom we conclude that

$$
a_{j k}(\lambda)=(-1)^{k} \frac{\Gamma\left(\frac{\lambda+j-k}{2}+1\right)}{\Gamma\left(\frac{-\lambda+j-k}{2}\right)} a_{j k}(-2-\lambda), \quad j, k \in \mathbb{N}_{0}, \lambda \in \mathbb{C} \backslash \mathbb{Z} .
$$

Note that the factor in (5.2) in front of $a_{j k}(-2-\lambda)$ is symmetric in $j$ and $k$.

We observe that the special case of $j=k$ in (5.2) was already given in [8, p. 782] and that formula (5.2) implies $a_{j k}(-1)=0$ if the minimum of $j$ and $k$ is odd, compare the remark following Proposition 2. Also note that formula (5.2) is a consequence of formula (2.2) and of the fact that $W_{\sigma}$ and $W_{\mathcal{F} \sigma}$ are closely connected.

Proposition 3 Let $\sigma \in \mathcal{S}^{\prime}\left(\mathbb{R}^{2}\right)$ be homogeneous on $\mathbb{R}^{2}$ of degree $\lambda \in \mathbb{C}$ and such that $W_{\sigma}$ is bounded on $L^{2}(\mathbb{R})$. Then $-2 \leq \operatorname{Re} \lambda \leq 0$ and [either $\sigma$ is a multiple of $\delta$ or $\sigma=F \cdot r^{\lambda}$ with $F \in \mathcal{H}^{(1 / 4+\mu / 2)}\left(\mathbb{S}^{1}\right)$ for $\left.\mu=\operatorname{Re} \lambda\right]$.

Proof (a) Let us first settle the case of homogeneous $\sigma$ with support contained in $\{0\}$. Then $\lambda=-2-j, j \in \mathbb{N}_{0}$, and $\sigma$ is a linear combination of $\partial_{x}^{k} \partial_{y}^{l} \delta$ for $k, l \in \mathbb{N}_{0}$ with $k+l=j$. This implies that $W_{\sigma}$ is a linear combination of the operators $A_{k l}$, $k+l=j$, where $\left(A_{k l} \phi\right)(x)=x^{k} \phi^{(l)}(-x)$ for $\phi \in \mathcal{S}(\mathbb{R})$. Therefore, $W_{\sigma}$ is bounded in this case if and only if $j=0$. Indeed, for $\phi \in \mathcal{S}(\mathbb{R})$, we have

$$
\lim _{N \rightarrow \infty} N^{-k}\left\|A_{k l}(\phi(x+N))\right\|_{2}=\left\|\phi^{(l)}\right\|_{2}
$$

and the boundedness of $A_{k l}$ implies $k=0$, and

$$
\left\|A_{0 l}(\phi(N x))\right\|_{2}=N^{l-1 / 2}\left\|\phi^{(l)}\right\|_{2}, \quad N>0
$$

and the boundedness of $A_{0 l}$ implies $l=0$ and hence $\sigma$ is a multiple of $\delta$.

(b) Let now $\sigma=F \cdot r^{\lambda}$ with $F(\omega)=\sum_{k \in \mathbb{Z}} c_{k} \omega^{k} \in \mathcal{D}^{\prime}\left(\mathbb{S}^{1}\right)$ and assume that $c_{m} \neq 0$ for some $m \in \mathbb{Z}$. In particular, in the case of $\lambda=-2-j, j \in \mathbb{N}_{0}$, the homogeneity of $\sigma$ on $\mathbb{R}^{2}$ implies that $|m|>j$. 
Since $W_{\sigma} \in \mathcal{L}\left(L^{2}(\mathbb{R})\right)$, its matrix coefficients $b_{j k}=\left\langle e_{j}, W_{\sigma} e_{k}\right\rangle$ are bounded in modulus by $\left\|W_{\sigma}\right\|_{\mathcal{L}\left(L^{2}(\mathbb{R})\right)}$. Hence also the complex sequence $a_{k, k+m}(\lambda)=$ $b_{k, k+m} / c_{m}, k \in \mathbb{N}_{0}, k \geq-m$, must be bounded.

Let us next determine the asymptotic behavior of $a_{k+m, k}(\lambda)$ for $k \rightarrow \infty$ and $m \in \mathbb{N}_{0}$ fixed, and for $\lambda \in \mathbb{C} \backslash\left(-2-\mathbb{N}_{0}\right)$ or such that $\lambda=-2-j, j \in \mathbb{N}_{0}$, and $m>j$.

Upon inserting the standard integral representation for the hypergeometric function (see [5, Equ. 9.111]) into formula (5.1) and using analytic continuation, we obtain

$a_{k+m, k}(\lambda)=2^{m / 2} \sqrt{\frac{(k+m) !}{k !}}\left\langle\frac{t_{+}^{(m-\lambda) / 2-1}}{\Gamma\left(\frac{m-\lambda}{2}\right)} \cdot(1-t)_{+}^{(m+\lambda) / 2},(1-2 t)^{k}\right\rangle, k, m \in \mathbb{N}_{0}$.

Note that $t_{+}^{(m-\lambda) / 2-1} / \Gamma\left(\frac{m-\lambda}{2}\right)$ is an entire distribution-valued function of $\lambda$ which can be multiplied with $(1-t)_{+}^{(m+\lambda) / 2}$ since the singular supports of the two distributions are disjoint. Also note that $(1-t)_{+}^{(m+\lambda) / 2}$ is analytic at the value of $\lambda$ which we consider.

We then obtain the asymptotic behavior of $a_{k+m, k}(\lambda)$ for $k \rightarrow \infty$ by approximating $(1-2 t)^{k}$ in formula (5.3) by $(1-t)^{2 k}$ near zero and by $\left(-t^{2}\right)^{k}$ near one, respectively. This implies

$$
\begin{aligned}
a_{k+m, k}(\lambda) & \sim 2^{m / 2} \sqrt{\frac{(k+m) !}{k !}}\left[\frac{\Gamma\left(\frac{m+\lambda}{2}+2 k+1\right)}{\Gamma(m+2 k+1)}+(-1)^{k} \frac{\Gamma\left(\frac{m-\lambda}{2}+2 k\right) \Gamma\left(\frac{m+\lambda}{2}+1\right)}{\Gamma\left(\frac{m-\lambda}{2}\right) \Gamma(m+2 k+1)}\right] \\
& \sim(2 k)^{m / 2}\left[(2 k)^{(\lambda-m) / 2}+(-1)^{k} \frac{\Gamma\left(\frac{m+\lambda}{2}+1\right)}{\Gamma\left(\frac{m-\lambda}{2}\right)}(2 k)^{-(m+\lambda+2) / 2}\right] .
\end{aligned}
$$

Hence, eventually,

$$
a_{k+m, k}(\lambda) \sim \begin{cases}(2 k)^{\lambda / 2} & : \operatorname{Re} \lambda>-1, \\ (2 k)^{\lambda / 2}+(-1)^{k} \frac{\Gamma\left(\frac{m+\lambda}{2}+1\right)}{\Gamma\left(\frac{m-\lambda}{2}\right)}(2 k)^{-\lambda / 2-1} & : \operatorname{Re} \lambda=-1, \\ (-1)^{k} \frac{\Gamma\left(\frac{m+\lambda}{2}+1\right)}{\Gamma\left(\frac{m-\lambda}{2}\right)}(2 k)^{-\lambda / 2-1} & : \operatorname{Re} \lambda<-1,\end{cases}
$$

for $k \rightarrow \infty$ and $m \in \mathbb{N}_{0}$ with $m>|2+\lambda|$ if $\lambda \in-2-\mathbb{N}_{0}$. Here the precise meaning of $v_{k} \sim w_{k}$ is $\lim _{k \rightarrow \infty} k^{-c}\left(v_{k}-w_{k}\right)=0$ for $c=\frac{1}{2} \max (\operatorname{Re} \lambda,-2-\operatorname{Re} \lambda)$. We remark that the special case of $m=0$ was shown already in [8, p. 783].

According to the above, if $c_{m} \neq 0$ for some $m \in \mathbb{N}_{0}$, then the boundedness of $a_{k+m, k}(\lambda)$ implies $\operatorname{Re} \lambda \in[-2,0]$ due to formula (5.4). If instead $c_{m} \neq 0$ for $m<0$, then we use the boundedness of $a_{k, k+m}(\lambda)=a_{l+|m|, l}(\lambda)$ for $l=k+m$ with $k \geq|m|$. (c) Let us finally show that $F \in \mathcal{H}^{(1 / 4+\mu / 2)}\left(\mathbb{S}^{1}\right)$ for $\mu=\operatorname{Re} \lambda$. First we observe that

$$
a_{j 0}(\lambda)=2^{j / 2} \frac{\Gamma\left(\frac{\lambda+j}{2}+1\right)}{\sqrt{j !}}=\sqrt[4]{\pi} \sqrt{\frac{\Gamma\left(\frac{\lambda+j}{2}+1\right)^{2}}{\Gamma\left(\frac{j+1}{2}\right) \Gamma\left(\frac{j}{2}+1\right)}} \sim \sqrt[4]{\pi}\left(\frac{j}{2}\right)^{1 / 4+\lambda / 2}, \quad j \rightarrow \infty,
$$


according to [5, Equ. 8.328.2]. If $e_{j}, j \in \mathbb{N}_{0}$, denotes, as before, the orthonormal basis of normalized Hermite functions, see formula (3.1), we infer therefore from

$$
W_{\sigma} e_{0}=\sum_{j=0}^{\infty} e_{j} b_{j 0}=\sum_{j=0}^{\infty} e_{j} a_{j 0} c_{-j}
$$

and from $W_{\sigma} \in \mathcal{L}\left(L^{2}(\mathbb{R})\right)$ that $\left(a_{j 0} c_{-j}\right)_{j \in \mathbb{N}_{0}} \in l^{2}\left(\mathbb{N}_{0}\right)$ and hence that also the sequence $\left(c_{-j}(1+j)^{1 / 4+\mu / 2}\right)_{j \in \mathbb{N}_{0}}$ belongs to $l^{2}\left(\mathbb{N}_{0}\right)$.

Similarly, if $\phi \in s\left(\mathbb{N}_{0}\right)$ with $\|\phi\|_{l^{2}\left(\mathbb{N}_{0}\right)} \leq 1$, then

$$
\left\|W_{\sigma}\right\|_{\mathcal{L}\left(L^{2}(\mathbb{R})\right)} \geq\left|\left\langle W_{\sigma}\left(\sum_{k \in \mathbb{N}_{0}} \phi_{k} e_{k}\right), e_{0}\right\rangle\right|=\left|\sum_{k=0}^{\infty} a_{0 k} c_{k} \phi_{k}\right|
$$

and thus $\left(c_{k}(1+k)^{1 / 4+\mu / 2}\right)_{k \in \mathbb{N}_{0}} \in l^{2}\left(\mathbb{N}_{0}\right)$. Altogether we conclude that $F$ belongs to the Sobolev space $\mathcal{H}^{(1 / 4+\mu / 2)}\left(\mathbb{S}^{1}\right)$ and this completes the proof.

Remark Let us yet comment on the situation for symbols $\sigma$ that are homogeneous on $\mathbb{R}^{2} \backslash\{0\}$, but only associated homogeneous on $\mathbb{R}^{2}$, i.e., $\sigma=F \cdot r^{-2-j}, j \in \mathbb{N}_{0}$, and $F(\omega)=\sum_{k \in \mathbb{Z}} c_{k} \omega^{k}$ with $c_{k} \neq 0$ for some $k \in \mathbb{Z}$ with $|k| \leq j$. In this case, $W_{\sigma}$ cannot be bounded on $L^{2}(\mathbb{R})$. We have omitted this case in Proposition 3 since the asymptotic expansion in formula (5.4) becomes slightly more complicated. As to be expected, logarithms appear. Just to get an idea, let us analyze the case of homogeneity $\lambda=-2$.

According to the complement formula (5.2), we obtain

$$
a_{j k}(-2)=(-1)^{\min (j, k)} \cdot \frac{2 a_{j k}(0)}{|j-k|}, \quad j \neq k \in \mathbb{N}_{0} .
$$

In particular, this implies

$$
\exists C>0: \forall j \neq k \in \mathbb{N}_{0}:\left|a_{j k}(-2)\right| \leq C(1+|j-k|)^{-3 / 4}
$$

and hence $W_{\sigma} \in \mathcal{L}\left(L^{2}(\mathbb{R})\right)$ if $\sigma=F \cdot r^{-2}, F=\sum_{k \in \mathbb{Z}} c_{k} \omega^{k}, \sigma$ is homogeneous on $\mathbb{R}^{2}$, i.e., $c_{0}=0$, and $\sum_{k \in \mathbb{Z}}\left|c_{k}\right|(1+|k|)^{-3 / 4}<\infty$.

On the other hand, $a_{j j}(-2)$ grows logarithmically for $j \rightarrow \infty$ and thus $W_{\sigma}$ is not bounded if $\sigma=r^{-2}=\operatorname{Pf}_{\lambda=-2} r^{\lambda}$. In fact, setting $m=0$ and $k=j \in \mathbb{N}$ in formula (5.3) yields

$$
\begin{aligned}
a_{j j}(-2) & =\operatorname{Pf}_{\lambda=-2} a_{j j}(\lambda)=\operatorname{Pf}_{z=0} a_{j j}(-2-2 z) \\
& =\left\langle\operatorname{Pf}_{z=0}\left(\frac{t_{+}^{z}}{\Gamma(1+z)} \cdot(1-t)_{+}^{-1-z}\right),(1-2 t)^{j}\right\rangle \\
& =\left\langle\left.\frac{\mathrm{d}}{\mathrm{d} z}\left(\frac{t_{+}^{z}}{\Gamma(1+z)}\right)\right|_{z=0} \cdot \operatorname{Res}_{z=0}(1-t)_{+}^{-1-z}+Y(t)(1-t)_{+}^{-1},(1-2 t)^{j}\right\rangle
\end{aligned}
$$




$$
\begin{aligned}
& =\left\langle(\psi(1)-\log t) \cdot \delta(1-t)+Y(t)(1-t)_{+}^{-1},(1-2 t)^{j}\right\rangle \\
& =\psi(1)(-1)^{j}+\int_{0}^{1} \frac{(1-2 t)^{j}-(-1)^{j}}{1-t} \mathrm{~d} t \\
& =(-1)^{j}\left[\psi(1)+2 j \int_{0}^{1}(1-2 s)^{j-1} \log s \mathrm{~d} s\right] \\
& =(-1)^{j}\left[\psi(1)+2 j \int_{0}^{1}(1-s)^{2 j-2} \log s \mathrm{~d} s+o(1)\right] \\
& =2(-1)^{j+1}[\psi(2 j)-\psi(1)]+o(1) .
\end{aligned}
$$

Hence $a_{j j}(-2)=2(-1)^{j+1}[\log (2 j)-\psi(1)]+o(1)$ for $j \rightarrow \infty$. (Here the terms $o(1)$ denote series converging to 0$)$.

\section{A sufficient condition ensuring the boundedness of $W_{\sigma}$}

When comparing the three examples $\lambda=0$ (in Proposition 2), $\lambda=-1$ (in the remark following Proposition 2) and $\lambda=-2$ (in the remark following Proposition 3 ), we see that we have to stipulate an $l^{1}$ condition on the Fourier coefficients $c_{k}$ of the characteristic $F$ of the symbol $\sigma=F \cdot r^{\lambda}$ in order to guarantee that $W_{\sigma}$ is bounded. The following proposition incorporates these special cases into a general statement.

Proposition 4 Let $F(\omega)=\sum_{k \in \mathbb{Z}} c_{k} \omega^{k} \in \mathcal{D}^{\prime}\left(\mathbb{S}^{1}\right)$ and $\lambda \in \mathbb{C}$ such that $\mu=\operatorname{Re} \lambda \in$ $[-2,0]$ and assume that $\sigma=F \cdot r^{\lambda}$ is homogeneous on $\mathbb{R}^{2}$. (This just means that $c_{0}$ vanishes if $\lambda=-2$.) Then the following holds:

If $\sum_{k \in \mathbb{Z}}\left|c_{k}\right|(1+|k|)^{1 / 4+\mu / 2}<\infty$, then $W_{\sigma}$ is a bounded operator on $L^{2}(\mathbb{R})$.

Proof (a) We have dealt with the special case of $\lambda=-2$ in the remark following Proposition 3. We can therefore assume that $\lambda \neq-2$. According to condition (3.5) in Proposition 1, we then have to verify the following estimate:

$$
\exists C>0: \forall j, k \in \mathbb{N}_{0}:\left|a_{j k}(\lambda)\right| \leq C(1+|j-k|)^{1 / 4+\mu / 2} .
$$

Note that the asymptotic expansion in formula (5.4) already shows that $a_{j j}(\lambda), j \in \mathbb{N}_{0}$, is a bounded sequence. Therefore it suffices to prove the estimate in (6.1) for $j>k$. (b) Let us define now

$$
\tilde{a}_{j k}(\lambda):=\int_{0}^{\infty} \frac{(1-s)^{k}}{(1+s)^{j+1}} s^{(j-k-\lambda) / 2-1} \mathrm{~d} s, \quad j, k \in \mathbb{N}_{0}, j>k .
$$

We point out that the integral in (6.2) is absolutely convergent for $\mu=\operatorname{Re} \lambda \in[-2,0]$.

Due to [5, Equs. 3.197.1 and 9.131.1] the integral in (6.2) yields the following:

$$
\tilde{a}_{j k}(\lambda)=\frac{\Gamma\left(\frac{j-k-\lambda}{2}\right) \Gamma\left(\frac{\lambda+j-k}{2}+1\right)}{(j-k) !} \cdot{ }_{2} F_{1}\left(-k, \frac{j-k-\lambda}{2} ; j-k+1,2\right) .
$$


Combining formulas (5.1) and (6.3), we obtain

$$
a_{j k}(\lambda)=2^{(j-k) / 2} \sqrt{\frac{j !}{k !}} \cdot \frac{\tilde{a}_{j k}(\lambda)}{\Gamma\left(\frac{j-k-\lambda}{2}\right)}, \quad j, k \in \mathbb{N}_{0}, j>k, \mu=\operatorname{Re} \lambda \in[-2,0] .
$$

(c) Let us estimate $\tilde{a}_{j k}(\lambda)$ for $j>k, \mu=\operatorname{Re} \lambda \in[-2,0]$. By dividing the range of integration we obtain

$$
\begin{aligned}
\tilde{a}_{j k}(\lambda) & =\int_{0}^{1} \frac{(1-s)^{k}}{(1+s)^{j+1}} s^{(j-k-\lambda) / 2-1} \mathrm{~d} s+\int_{0}^{1} \frac{\left(1-s^{-1}\right)^{k}}{\left(1+s^{-1}\right)^{j+1}} s^{-(j-k-\lambda) / 2-1} \mathrm{~d} s \\
& =\int_{0}^{1} \frac{(1-s)^{k}}{(1+s)^{j+1}} s^{(j-k) / 2-1}\left[s^{-\lambda / 2}+(-1)^{k} s^{\lambda / 2+1}\right] \mathrm{d} s
\end{aligned}
$$

and hence

$$
\left|\tilde{a}_{j k}(\lambda)\right| \leq 2 \int_{0}^{1} \frac{(1-s)^{k}}{(1+s)^{j+1}} s^{(j-k) / 2-1} \mathrm{~d} s .
$$

The standard integral representation [5, Equ. 9.111] of the hypergeometric function therefore yields

$$
\left|\tilde{a}_{j k}(\lambda)\right| \leq 2 \frac{\Gamma\left(\frac{j-k}{2}\right) k !}{\Gamma\left(\frac{j+k}{2}+1\right)} \cdot{ }_{2} F_{1}\left(j+1, \frac{j-k}{2} ; \frac{j+k}{2}+1 ;-1\right) .
$$

Upon inserting [2, Equ. 7.3.6.1] we obtain the following estimate of $\left|\tilde{a}_{j k}(\lambda)\right|$ by an upper bound independent of $\lambda$ and valid for $j>k, \mu=\operatorname{Re} \lambda \in[-2,0]$ :

$$
\left|\tilde{a}_{j k}(\lambda)\right| \leq 2^{-j} \Gamma\left(\frac{j-k}{2}\right) k ! \sqrt{\pi}\left[\frac{1}{\Gamma\left(\frac{j}{2}+1\right) \Gamma\left(\frac{k+1}{2}\right)}+\frac{1}{\Gamma\left(\frac{j+1}{2}\right) \Gamma\left(\frac{k}{2}+1\right)}\right] .
$$

(d) Combining formulas (6.4) and (6.5) and using the duplication formula for the gamma function yields, still for $j>k, \mu=\operatorname{Re} \lambda \in[-2,0]$,

$$
\left|a_{j k}(\lambda)\right| \leq \frac{\Gamma\left(\frac{j-k}{2}\right)}{\left|\Gamma\left(\frac{j-k-\lambda}{2}\right)\right|}\left(\frac{\alpha_{j}}{\alpha_{k}}+\frac{\alpha_{k}}{\alpha_{j}}\right)
$$

Here $\alpha_{j}=\sqrt{\Gamma\left(\frac{j}{2}+1\right) / \Gamma\left(\frac{j+1}{2}\right)}$ is defined as in Proposition 2. The limit [5, Equ. 8.328.2] implies

$$
\exists C>0: \forall j, k \in \mathbb{N}_{0} \text { with } j \geq k: \frac{\Gamma\left(\frac{j-k}{2}\right)}{\left|\Gamma\left(\frac{j-k-\lambda}{2}\right)\right|} \leq C(1+j-k)^{\mu / 2}
$$


with $\mu=\operatorname{Re} \lambda$. Hence the estimate of $\alpha_{j} / \alpha_{k}$ in formula (4.6) and the monotonicity of $\alpha_{j}$ imply

$$
\exists C>0: \forall j, k \in \mathbb{N}_{0} \text { with } j>k:\left|a_{j k}(\lambda)\right| \leq C(1+j-k)^{1 / 4+\mu / 2} .
$$

Therefore, the estimate (6.1) holds in generality and this completes the proof.

Remark Let us finally compare the assumption

$$
F \in L^{2}\left(\mathbb{S}^{1}\right) \text {, i.e., }\left(c_{k}\right)_{k \in \mathbb{Z}} \in l^{2}(\mathbb{Z})
$$

with the hypothesis made in Proposition 4, namely

$$
\left((1+|k|)^{1 / 4+\mu / 2} c_{k}\right)_{k \in \mathbb{Z}} \in l^{1}(\mathbb{Z}) .
$$

At the end of Sect. 2, we have seen that in the case of $-2<\mu=\operatorname{Re} \lambda<-1$, assumption (A) implies the boundedness of $W_{\sigma}$ on $L^{2}(\mathbb{R})$ for $\sigma=F \cdot r^{\lambda}$. Instead, assumption (B) implies that $W_{\sigma}$ is bounded whenever $\mu \in[-2,0]$ according to Proposition 4.

If $-2<\mu<-\frac{3}{2}$, then $\left((1+|k|)^{1 / 4+\mu / 2}\right)_{k \in \mathbb{Z}} \in l^{2}(\mathbb{Z})$ and hence (A) implies (B). So condition (B) is the less restrictive and more general one. In contrast, when $-\frac{3}{2} \leq \mu<-1$, then neither of the two assumptions (A) and (B) implies the other one. In fact, e.g., for $\mu=-\frac{3}{2}$ and $c_{k}=(1+|k|)^{-1 / 2} / \log (1+|k|)$, condition (A) holds whereas (B) fails. Conversely, if $\mu=-\frac{3}{2}$ and $c_{k}=2^{j / 2} j^{-2}$ for $k=2^{j}, j \in \mathbb{N}$, and $c_{k}=0$ for $k \notin\left\{2^{j} ; j \in \mathbb{N}\right\}$, then

$$
\sum_{k \in \mathbb{Z}}(1+|k|)^{-1 / 2}\left|c_{k}\right|=\sum_{j=1}^{\infty}\left(1+2^{j}\right)^{-1 / 2} 2^{j / 2} j^{-2} \leq \sum_{j=1}^{\infty} j^{-2}<\infty
$$

but $\left(c_{k}\right)_{k \in \mathbb{Z}} \notin l^{2}(\mathbb{Z})$. Hence (B) holds and (A) fails.

As was pointed out already in the introduction, in contrast to the case of radially symmetric distributions, we cannot hope for a condition that is both necessary and sufficient for the boundedness of $W_{\sigma}$ if $\sigma$ is a tensor product in polar coordinates. This is a consequence of the fact that the Hermite functions in this case do not yield eigenfunctions of $W_{\sigma}$. So the approach in Proposition 1 has its limitations from the word go.

Acknowledgements Open access funding provided by University of Innsbruck and Medical University of Innsbruck. I am very grateful to Norbert Ortner for his continuous interest in the subject of this paper and our many discussions on it. Without him, this paper definitely would not have come into existence. (Whether this had not meant any loss to the mathematical world or whether, perhaps, somebody else will take an interest in this little study is a question I do not venture to bet on).

Open Access This article is licensed under a Creative Commons Attribution 4.0 International License, which permits use, sharing, adaptation, distribution and reproduction in any medium or format, as long as you give appropriate credit to the original author(s) and the source, provide a link to the Creative Commons licence, and indicate if changes were made. The images or other third party material in this article are included 
in the article's Creative Commons licence, unless indicated otherwise in a credit line to the material. If material is not included in the article's Creative Commons licence and your intended use is not permitted by statutory regulation or exceeds the permitted use, you will need to obtain permission directly from the copyright holder. To view a copy of this licence, visit http://creativecommons.org/licenses/by/4.0/.

\section{References}

1. Badii, L., Oberhettinger, F.: Tables of Laplace Transforms. Springer, New York (1973)

2. Brychkov, Yu.A., Marichev, O.I., Prudnikov, A.P.: Integrals and Series, vol. 3. More Special Functions. Gordon \& Breach, New York (1990); Translated from А.П. Прудников, Ю.А. Брычков и О.И. Маричев: Интегралы и Ряды, дополнительные главы. Наука, Москва (1986)

3. Folland, G.B.: Harmonic Analysis in Phase Space. Annals of Mathematics Studies, vol. 122. Princeton University Press, Princeton (1989)

4. Friedlander, G., Joshi, M.: Introduction to the Theory of Distributions, 2nd edn. Cambridge University Press, Cambridge (1998)

5. Gradshteyn, I.S., Ryzhik, I.M.: Table of Integrals, Series and Products. Academic Press, New York (1980); Translated from И. С. Градштейн и И. М. Рыжик: Таблицы Интегралов, Сумм, Рядов и Произведений. Наука, Москва (1971)

6. Ortner, N., Wagner, P.: Distribution-Valued Analytic Functions. Tredition, Hamburg (2013)

7. Ortner, N., Wagner, P.: Fundamental Solutions of Linear Partial Differential Operators. Springer, New York (2015)

8. Ortner, N., Wagner, P.: On the Weyl transform for rotationally invariant symbols. J. Pseudo-Differ. Oper. Appl. 10, 769-791 (2019)

9. Schwartz, L.: Théorie des Distributions, 2nd edn. Hermann, Paris (1966)

10. Stein, E.M., Weiss, G.: Introduction to Fourier Analysis on Euclidean Spaces. Princeton University Press, Princeton (1971)

11. Wong, M.W.: Weyl Transforms. Springer, New York (1998)

Publisher's Note Springer Nature remains neutral with regard to jurisdictional claims in published maps and institutional affiliations. 